\author{
MS40-O2 Microsecond-resolved in-situ \\ SAXS/WAXS experiments on the \\ nucleation and growth of CdS quantum dots \\ along a free liquid jet \\ Andreas Magerl ${ }^{1}$, Andreas Schiener ${ }^{1}$
}

1. Friedrich-Alexander University of Erlangen-Nuremberg; Crystallography and Structural Physics; Staudtstr. 3; 91058 Erlangen; Germany

\section{email: andreas.magerl@fau.de}

The tremendously growing scientific and industrial interest on semiconducting nanoparticles (quantum dots) relates to the change of the physical and chemical properties of a semiconductor, when its size becomes smaller than the exciton diameter of the bulk material. This so called quantum confinement effect allows tailoring particles properties by controlling its size. To manufacture suitable particles, it is important to get a detailed understanding of the undelying nucleation and growth machnisms. With the present study we pioneer a novel method to measure both the morphology (SAXS) and the crystalline structure (WAXS) during the early stages of particle formation via fast in-situ experiments along a free liquid jet. Diluted chemicals are brought together in a Y-shaped micro mixer and ejected as a continuous free jet after mixing. The nanoparticle formation takes place in the free jet and different formation stages are adressed by targeting a monochromatic synchrotron X-ray beam on different positions along the jet. This allows to access reaction times between $10 \mu \mathrm{s}$ and $10 \mathrm{~ms}$, while illuminating these stages for exposure times up to several seconds in order to obtain a high quality scattering signal. We have investigated the nucleation and early growth of $\mathrm{CdS}$ without disturbing the reaction by the addition of stabilizers. These experiments give for the first time a detailed insight into the nucleation and growth process of nanoparticles by precipitation reaction on a time scale which is tree orders of magnitude smaller than in earlier studies. The results indicate that the nucleation of $\mathrm{CdS}$ prenucleation clusters already takes place on the sub-ms time scale. Furthermore, the temperature and time-dependence of the quantum dot formation proof a diffusion limited growth mechanism for larger particles, which appears to be a very fundamental formation mechanism for these type of fast precipitation reactions.

Keywords: In-Situ; SAXS/WAXS; CdS; Quantum Dots
MS40-O3 Serial femtosecond

crystallography on in vivo grown crystals at SACLA - developments and results

Leonard M.G. Chavas ${ }^{1}$, Sasa Bajt ${ }^{2}$, Lars Gumprecht ${ }^{1}$, Henry N. Chapman ${ }^{1,3,4}$

1. Center for Free-Electron Laser Science, Deutsches Elektronen-Synchrotron DESY, Notkestraße 85, 22607 Hamburg, Germany

2. Photon Science, Deutsches Elektronen-Synchrotron DESY, Notkestraße 85, 22607 Hamburg, Germany

3. Department of Physics, University of Hamburg, Luruper Chaussee 149, 22607 Hamburg, Germany

4. Centre for Ultrafast Imaging, Luruper Chaussee 149, 22607 Hamburg, Germany

email: leonard.chavas@desy.de

Serial femtosecond crystallography (SFX) uses x-ray pulses from free-electron laser (FEL) sources to outrun radiation damages and thereby overcome long-standing limits in the structure determination of macromolecular crystals. Intense x-ray FEL pulses allow the collection of damage-free data at room temperature, and give the opportunity to study highly time-resolved reactions including irreversible events. By taking full advantage of high repetition rate x-ray pulse delivery schemes, protein structures could be determined in just minutes of measurement time. Automation in sample delivery during SFX experiments could therefore induce a turnover of samples much higher than at today's third generation synchrotrons and automated macromolecular crystallography beamlines, as no crystal alignment or complex robotic motions are required.

Challenges exist at various levels of the experiments, going from the vast nature of the samples to be screened at FELs, handling a large number of samples with minimum human intervention, but also fully characterising the overall injection and sample environment system. The Centre for Free-Electron Laser science has developed a scientific unit that concentrates on breaking down these various milestones and prepares ways for the SFX scientific community to experiment in a user friendly and reliable environment. The presentation will introduce the latest tools that were engineered for high-throughput SFX, emphasising on the developments for automated replenishment of samples and sample injection devices. Early results of SFX experiments on in vivo grown crystals performed at the Japanese XFEL SACLA will be highlighted.

Keywords: SFX, XFEL, in vivo crystals, high-throughput 